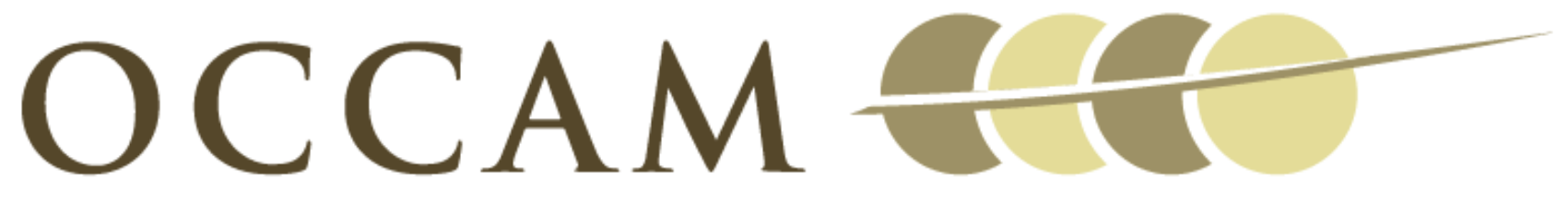

OXFORD CENTRE FOR COLLABORATIVE APPLIED MATHEMATICS

Report Number 11/20

Hybrid modelling of individual movement and collective behaviour by

Benjamin Franz and Radek Erban

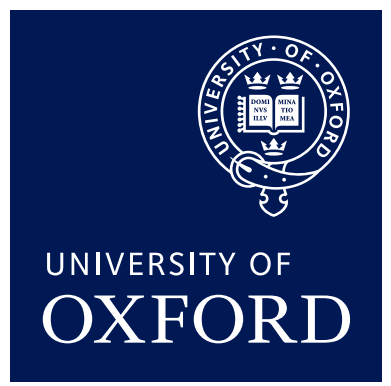

Oxford Centre for Collaborative Applied Mathematics Mathematical Institute 24 - 29 St Giles'

Oxford

OX1 3LB

England 



\title{
Hybrid modelling of individual movement and collective behaviour
}

\author{
Benjamin Franz and Radek Erban
}

\begin{abstract}
Mathematical models of dispersal in biological systems are often written in terms of partial differential equations (PDEs) which describe the time evolution of population-level variables (concentrations, densities). A more detailed modelling approach is given by individual-based (agent-based) models which describe the behaviour of each organism. In recent years, an intermediate modelling methodology - hybrid modelling - has been applied to a number of biological systems. These hybrid models couple an individual-based description of cells/animals with a PDEmodel of their environment. In this chapter, we overview hybrid models in the literature with the focus on the mathematical challenges of this modelling approach. The detailed analysis is presented using the example of chemotaxis, where cells move according to extracellular chemicals that can be altered by the cells themselves. In this case, individual-based models of cells are coupled with PDEs for extracellular chemical signals. Travelling waves in these hybrid models are investigated. In particular, we show that in contrary to the PDEs, hybrid chemotaxis models only develop a transient travelling wave.
\end{abstract}

\section{Introduction}

There are two fundamentally different approaches to the mathematical modelling of systems of interacting individuals (cells, animals) in biology. If the number of individuals is large, one often uses a continuum population-level approach, which yields partial differential equations (PDEs) for the spatially-distributed densities of individuals [35]. The advantage of PDE-based modelling is a well-developed mathematical theory and a number of existing numerical solvers which can be used to efficiently simulate the system behaviour. However, continuum approximation becomes inaccurate if smaller groups of individuals are studied, and agent-based (individual-

Mathematical Institute, University of Oxford, 24-29 St Giles', Oxford OX1 3LB, United Kingdom; e-mail: franz@maths.ox.ac.uk, erban@maths.ox.ac.uk 
based) models become the method of choice [10,44]. Examples can be found in zoological applications, like behaviour of fish schools, bird flocks and locust groups $[9,45]$. The individual behaviour of the agents is modelled as well as the interaction (e.g. attraction or repulsion) between them [11]. A number of these agents are then simulated on the computer and their collective behaviour is analysed. This approach allows for a more detailed description of the individual behaviour and does not discount various stochastic effects caused by a finite number of individuals. On the other hand mathematical analysis is often hard to achieve and simulations can be computationally intensive.

Another problem with purely agent-based models is that it is challenging to incorporate influences the agents might have on their environment. This is important whenever agents interact indirectly by modifying their (evolving) environment. A classical example is modelling chemotaxis where individual cells modify (secrete, consume) extracellular chemical signals which diffuse in the extracellular space $[12,18]$. In this case, a hybrid modelling framework that seeks to combine the advantages of continuum and agent-based models is often used. The main idea of this modelling approach is to describe some species as a continuum and some species as a set of agents. For example, Dallon and Othmer [12] developed a hybrid model for chemotaxis of slime mold Dictyostelium discoideum in which the cells are treated as individuals in a continuum field of the chemoattractant which evolves according to a reaction-diffusion PDE. A similar hybrid modelling framework has also been applied to chemotaxis of bacteria $[13,51]$ and leukocytes [22]. The use of the hybrid approach allows for faster simulations than the purely agent-based model which would treat extracellular chemicals as another set of agents. Extracellular signalling molecules are much smaller and more abundant than cells. This property is often used to justify that extracellular chemicals can be described as a continuum [12].

The use of hybrid models is becoming more widespread especially with the growing computational power that allows to consider more complex systems in this manner, including modelling tumour growth [39] and forest dynamics [33]. In cancer biology, several hybrid cellular automaton models have been proposed in the literature [40, 41]. For example, Smallbone et al. [41] coupled a two-dimensional cellular automaton model (describing cells) with continuum (PDE-based models) of glucose, $\mathrm{H}^{+}$and oxygen concentrations, building on the previous work of Patel et al. [39] and Alarcón et al. [3]. A similar hybrid approach has been used in a number of other studies in cancer biology [4, 21, 36]. A hybrid forest model with trees modelled as agents and a continuum approach used for oxygen and other atmospheric gases is presented in [33]. In economical research hybrid models are used to estimate prices in the petrol market [25] and in general markets with a non uniform spatial demand of products [26, 27]. In these models the demand is described as a continuous function of space whereas the retailers are considered as agents.

The term hybrid modelling is sometimes applied for models which use both individual-based and continuum description for the same physical quantity. For example, a "hybrid" model for the spread of an epidemic disease is presented in [8]. It initially considers infected individuals as agents, but switches to a continuum model when the number of infected people in an area rises above a threshold. Coupling 
reaction-diffusion models with a different level of detail in different parts of the computational domain is presented in $[15,19]$. "Hybrid" models of this type are useful because they can lead to computational savings. However, in this chapter, we will focus on hybrid models which describe some system components (e.g. cells or animals) as individual agents and some components (e.g. external chemicals) as continuum fields. The choice which description is used for each species is made at the beginning and will not change during the course of the simulation. We will summarise the progress in hybrid models which satisfy this definition, and clarify some of the problems and difficulties that arise from their use.

The outline of this chapter is as follows. Section 2 will give a short overview of the PDE-based and agent-based modelling approaches before the general mathematical framework for hybrid models is introduced in Section 3. Hybrid models can be considered as extensions of (purely) agent-based models. Therefore, their computer implementation often forms an integral part of the model. We will discuss it in detail in Section 4 where we describe the numerical simulation of hybrid models drawing special attention to the different treatment of the continuum and the agent-based subsystems as well as the problem of matching the two parts. In order to give a more practical insight into the topic we will perform a case study of a hybrid chemotactic model in Section 5. This case study will also be used to show some qualitative and quantitative differences that can occur when using a hybrid model instead of the corresponding continuum model.

\section{Continuum vs. agent-based models}

Hybrid modelling is an intermediate approach between continuum (PDE-based) models and agent-based models of systems of interacting individuals. In this section we briefly review these common modelling approaches in mathematical terms. We will make use of our notation later in Section 3 when hybrid models are considered.

Continuum (mean-field) models give rules for the evolution of the spatially dependent concentration vector $\mathbf{c} \equiv \mathbf{c}(\mathbf{x}, t)$ where $\mathbf{x} \in \Omega \subset \mathbb{R}^{m}, m=1,2$ or 3 , and $t$ is the simulation time. The components of the vector $\mathbf{c}$ can be densities of individuals (cells, animals) and concentrations of extracellular signals. As the concentration vector $\mathbf{c}$ can change both with position $\mathbf{x}$ and time $t$, a general continuum model takes the form

$$
\frac{\partial \mathbf{c}}{\partial t}=\mathscr{L}(\mathbf{c}, \mathbf{x}, t) \quad \mathbf{x} \in \Omega,
$$

where $\mathscr{L}$ is an operator on $\mathbf{c}$, which in most practical cases will be a differential or integral operator. To uniquely describe the time evolution of (1), one also has to specify suitable initial and boundary conditions.

\section{Example 1. Keller-Segel model}

Continuum modelling is used in many areas of mathematical biology [35]. In chemotaxis modelling (which will be the subject of Section 5), a classical example of (1) is the Keller-Segel model of chemotaxis [31]. Here, $\Omega \subset \mathbb{R}$ and the vector 
c has two components, i.e. $\mathbf{c}=\left[c_{1}, c_{2}\right]=[n, S]$ where $n \equiv n(x, t)$ is the density of cells and $S \equiv S(x, t)$ is the concentration of the chemoattractant. The evolution equation (1) is a coupled system of two PDEs for $n$ and $S$ :

$$
\begin{aligned}
& \frac{\partial n}{\partial t}=D_{n} \frac{\partial^{2} n}{\partial x^{2}}-\frac{\partial}{\partial x}\left(n \chi(S) \frac{\partial S}{\partial x}\right), \\
& \frac{\partial S}{\partial t}=D_{S} \frac{\partial^{2} S}{\partial x^{2}}-k(S) n,
\end{aligned}
$$

where $D_{n}$ and $D_{S}$ are diffusion constants of cells and chemoattractant, respectively. The strength of chemotaxis is controlled by chemotactic sensitivity $\chi(S)$ and therefore by the concentration of substrate $S$ which is consumed by cells with the rate $k(S)$.

The applicability of continuum modelling depends on the number of particles in the studied system. In Example 1, the interacting "particles" are unicellular microscopic organisms $(n)$ and molecules of chemical signal $(S)$. As there are often more signalling molecules than cells, the validity of mean-field assumptions is dictated by the number of cells in the system. If the system only consists of a few cells, it is more accurate to use an individual-based approach which is introduced in the next section.

\subsection{Agent-based modelling}

In contrary to the continuum models the so-called agent-based models treat every particle as an individual that follows an inherent set of rules. This means in particular that individual behaviour and interactions between different agents account for the possibly complex behaviour of the system. Agent-based models are commonly used for systems with a small number of individuals that follow non-trivial behavioural rules, for example in modelling of collective animal behaviour [11] or human crowds in panic situations [23]. While continuum models have a welldeveloped mathematical theory, agent-based models are sometimes written as computer routines which are difficult to theoretically analyse. The literature also fails to agree on a general definition of an agent. In this chapter, we use a definition which is slightly adopted from [48] and used in [20].

Definition 1. An agent is a system that uses a fixed set of rules based on communication with other agents and information about the environment in order to change its internal state and fulfil its design objective.

This definition, however, is only a formal description, which now has to be put into a more rigorous context. Following from Definition 1, the mathematical description of an agent has to incorporate the behavioural rules of an agent as well as the possibility of communication between them. Therefore, we assume a finite number $N$ 
of agents numbered from 1 to $N$. In general $N$ can depend on time, taking into account birth or death of agents. We define the current state of an agent by its internal state variable $\mathbf{y}_{i}(t), i=1, \ldots, N$, which can describe its position, velocity and internal memory. It is this internal state and its time evolution that describes the rules of an agent. Since these agents represent different individuals, we assume that other agents generally have no means to access all internal state variables. In order to allow for communication between the agents, we define a set of external states $\mathbf{w}_{i}(t)$, which are observable by other agents. The observable states $\mathbf{w}_{i}(t)$ of every agent are in principle available to every other agent, which is ensured by creating the set of external states $\mathscr{X}$. The general agent-based model following these definitions then takes the form

$$
\begin{aligned}
\mathbf{y}_{i}(t+\Delta t) & =\mathbf{f}_{i}\left(\mathbf{y}_{i}(t), t, \Delta t, \mathscr{X}\right), & & i=1, \ldots, N, \\
\mathbf{w}_{i}(t) & =\mathbf{g}_{i}\left(\mathbf{y}_{i}(t)\right), & & i=1, \ldots, N, \\
\mathscr{X} & =\left\{\mathbf{w}_{1}, \ldots, \mathbf{w}_{N}\right\} . & &
\end{aligned}
$$

We can see that the evolution of $\mathbf{y}_{i}$ is given by the function $\mathbf{f}_{i}$, which notably depends on the time step $\Delta t$. This general description can entail discretised versions of ordinary differential equations (ODEs) as well as stochastic differential equations (SDEs). Additionally, agent-based systems that only change discretely can be written in the form (4)-(6).

We understand the external states of an agent merely as an observable representation of the internal states, which is why $\mathbf{w}_{i}(t)$ directly depends on $\mathbf{y}_{i}(t)$ through the function $\mathbf{g}_{i}$. The distinction between observable and non-observable states is often used to represent internal memories that cannot be perceived by other agents.

\section{Example 2. Animal behaviour}

Agent-based models have been successfully used for the modelling of collective animal behaviour [45]. Couzin et al. [11] showed that a relatively simplistic model can yield complex collective behaviour and can be used to model fish schools and bird flocks.

In this model, the internal states of an agent $\mathbf{y}_{i}$ are defined to be its position $\mathbf{x}_{i} \in \mathbb{R}^{m}(m=2,3)$ and its velocity $\mathbf{v}_{i} \in \mathbb{R}^{m}$. Since both the position and velocity of an agent potentially influence the motion of other agents, both are observable and hence $\mathbf{w}_{i}=\mathbf{y}_{i}=\left[\mathbf{x}_{i}, \mathbf{v}_{i}\right] \in \mathbb{R}^{2 m}$, which means that $\mathbf{g}_{i}=$ Id. The update rules $\mathbf{f}_{i}, i=1, \ldots, N$, in this example are equivalent for each agent and incorporate the different rules for the different zones in the model (zone of attraction, orientation and repulsion).

\section{Example 3. Chemotactic movement under a stationary signal}

A simple agent-based model for chemotaxis in one dimension can be written as follows [24]: the internal state $y_{i}(t)$ of an agent is defined as its current position in $\mathbb{R}$. Additionally, we assume that the signal $S(x)$ is fixed and there is no interaction between agents, hence no observable states are required. All agents start at some initial position $y_{0, i} \in \mathbb{R}$ and move according to the stochastic differential equation 


$$
\mathrm{d} y_{i}(t)=\chi(S) \frac{\partial S}{\partial x} \mathrm{~d} t+\sqrt{2 D_{n}} \mathrm{~d} W, \quad i=1, \ldots, N
$$

where $\chi(S)$ is the chemotactic sensitivity function introduced in Example $1, D_{n}$ is the diffusion constant of the bacteria and $\mathrm{d} W$ is the Wiener-process, also known as Brownian motion [29]. We can discretise (7) to obtain an update rule equivalent to (4) as follows

$$
y_{i}(t+\Delta t)=y_{i}(t)+\chi(S) \frac{\partial S}{\partial x} \Delta t+\sqrt{2 D_{n} \Delta t} \xi
$$

where $\xi$ is a normally distributed random variable with zero mean and unit variance. In the limit of infinitely many particles, this agent-based description is equivalent to the PDE (2), which is written for the density of cells. However, if we considered a time-evolving signal which is consumed by cells as in Example 1, a purely agentbased model would have to simulate the trajectories of all signal molecules. This would be computational intensive and a hybrid model which combines agent-based simulations with PDEs can then be used to optimize computational efficiency and accuracy.

\section{Hybrid modelling - theoretical framework}

Because of their hybrid nature the general framework for these models necessarily combines the two frameworks presented in Section 2. We define a vector of continuous variables $\mathbf{c}(\mathbf{x}, t)$ on a domain $\Omega \subset \mathbb{R}^{m}, m=1,2$ or 3 . The update rule for $\mathbf{c}$ is again governed by an operator $\mathscr{L}$, which now also depends on the current states of the agents. The $N$ agents are represented by their internal state variables $\mathbf{y}_{i}(t)$ and their set of observable states $\mathbf{w}_{i}(t)$ defined in (5). To allow interactions between the agents and the continuous variables c, the set of observable states $\mathscr{X}$ as defined in (6) is used. The update rules for the system are

$$
\begin{aligned}
\frac{\partial \mathbf{c}}{\partial t} & =\mathscr{L}(\mathbf{c}, \mathbf{x}, t, \mathscr{X}), & & \mathbf{x} \in \Omega, \\
\mathbf{y}_{i}(t+\Delta t) & =\mathbf{f}_{i}\left(\mathbf{y}_{i}(t), t, \Delta t, \mathscr{X}, \mathbf{c}\right), & & i=1, \ldots, N,
\end{aligned}
$$

where $\mathscr{X}$ is given in (6). In (8) we see that the agents can influence the continuous variables $\mathbf{c}$ through the set of observable states $\mathscr{X}$. Similarly, the behaviour of the agents can be altered by the continuous variables, as the operator $\mathbf{f}_{i}$ now also depends on c. Figure 1 shows a graphical representation of the hybrid model. It contains the $N$ agents represented by the internal states $\mathbf{y}_{i}$ on the left. Through the function $\mathbf{g}_{i}$ the observable states $\mathbf{w}_{i}$ are generated which then influence the update of the continuous variables c as well as the agents' behaviour themselves. We, however, encounter a problem in this definition, as the continuous variables are defined for every time $t$, while the internal agent states are only defined for discrete times. To overcome this problem we can consider (9) in the limit $\Delta t \rightarrow 0$, where it takes the general form of an SDE 


$$
\mathrm{d} \mathbf{y}_{i}=\mathbf{f}_{i}^{(1)}\left(\mathbf{y}_{i}(t), t, \mathscr{X}, \mathbf{c}\right) \mathrm{d} t+\mathbf{f}_{i}^{(2)}\left(\mathbf{y}_{i}(t), t, \mathscr{X}, \mathbf{c}\right) \mathrm{d} W,
$$

where $\mathbf{f}_{i}^{(1)}$ and $\mathbf{f}_{i}^{(2)}$ respectively represent the stochastic and the deterministic part of the SDE.

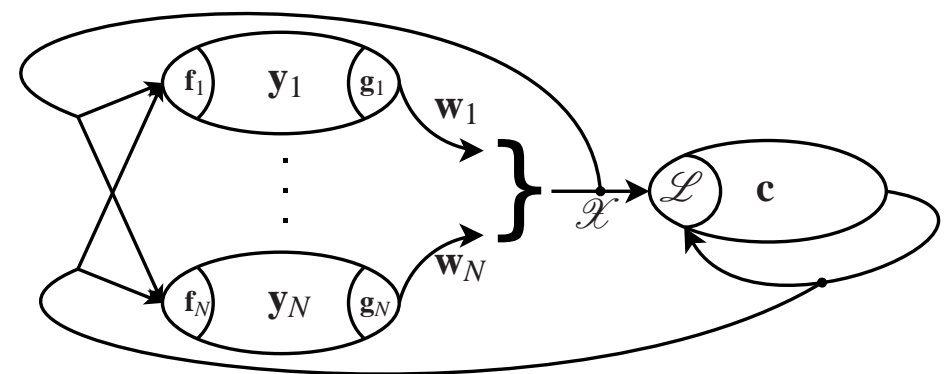

Fig. 1 Concept of a hybrid model. Arrows symbolise direction of influence.

\section{Example 4. Hybrid cellular automaton model for carcinogenesis}

In [41] Smallbone et al. present a hybrid cellular automaton model for the formation of cancer. This model uses reaction-diffusion equations to calculate the concentration of oxygen, glucose and hydrogen ions in the environment of the cells. The concentrations of these chemicals therefore constitute the continuous variables c. Each cell of the cellular automaton is represented by an agent with the internal state $y_{i} \in \mathbb{N}$ defining which of the finite number of possible phenotypes the cell at this position has (including the "phenotype" empty). As these phenotypes are observable by neighbouring cells, we have $w_{i}=y_{i}$. This cellular automaton model has a generation-based update rule, which means that the states $y_{i}$ are only updated once every time step. The rules of the model then represent the probabilistic functions $f_{i}$ in equation (4), where the change depends on the current phenotype, the neighbouring cells and the concentrations of the considered chemicals at the cell position.

\section{Example 5. Hybrid model for chemotaxis of Dictyostelium discoideum}

Dallon and Othmer developed a hybrid model for the chemotaxis of Dictyostelium discoideum [12] that combines individual cell movement with a continuous extracellular concentration of cAMP modelled by a PDE. The internal states of the agents are the position of the individual $\mathbf{x}_{i}$, as well as the variables representing the intracellular processes. Only the position and one of the intracellular variables influence the external field and therefore form the observable states $\mathbf{w}_{i}$. The update rules $\mathbf{f}_{i}$ are given through ODEs for the internal dynamics and rules of motion for the position. 


\subsection{A position-based hybrid model}

So far, we have defined a general framework for a hybrid model that allows for a great freedom in the choice of internal and external states of the agents. In the next step we want to refine this framework for the more specific models used in chemotaxis modelling $[12,17,43]$. In order to be able to interpret the agents as part of a species situated inside the domain $\Omega$, we need to introduce the notion of an agent's position in $\Omega$. Moreover, we assume that all agents are equal for an external observer except for their position, or in other words the set of observable states of the agents $\mathbf{w}_{i}(t)$ is the position $\mathbf{x}_{i}(t)$ of the agents inside $\Omega$, i.e.

$$
\mathbf{w}_{i}(t) \equiv \mathbf{x}_{i}(t) .
$$

This definition excludes Couzin et al. models for animal behaviour [11] as well as cellular automaton models [41], but it is sufficient for the chemotaxis example studied in Section 5.

Because of the agents' similarity, we no longer need to define an abstract set $\mathscr{X}$, but can instead define a density function $\rho_{\delta}$ on $\Omega$ through

$$
\rho_{\delta}(\mathbf{x}, t)=\sum_{i=1}^{N} \delta\left(\mathbf{x}-\mathbf{x}_{i}(t)\right), \quad \mathbf{x} \in \Omega
$$

When discussing numerical simulations of hybrid models, we will see that this definition of $\rho_{\delta}$ is already a first step towards obtaining a continuous density function for the agents. With this definition we can redefine the operator $\mathscr{L}$, which governs the behaviour of the continuous variables $\mathbf{c}$ and (1) reads as follows

$$
\frac{\partial \mathbf{c}}{\partial t}=\mathscr{L}\left(\mathbf{c}, \mathbf{x}, t, \rho_{\delta}\right)
$$

For the evolution of the internal agent states $\mathbf{y}_{i}$ we assume now that every agent can only perceive information about the continuous variables $\mathbf{c}$ at its current position. Hence, the operator $\mathbf{f}_{i}$ no longer depends on $\mathbf{c}$ on the whole domain, but only on $\mathbf{c}\left(\mathbf{x}_{i}\right)$ and the first spatial derivative in this point, i.e. $\mathbf{f}_{i}, i=1, \ldots, N$, are functions for all further considerations. Equation (9) therefore becomes

$$
\mathbf{y}_{i}(t+\Delta t)=\mathbf{f}_{i}\left(\mathbf{y}_{i}, t, \Delta t, \rho_{\delta}, \mathbf{c}\left(\mathbf{x}_{i}, t\right), \nabla \mathbf{c}\left(\mathbf{x}_{i}, t\right)\right) .
$$

This special type of hybrid systems still allows for a wide range of flexibility and can therefore be used to model a variety of different processes. In Section 5 we study position-based models for chemotaxis in more depth. 


\subsection{Initial and boundary conditions}

An important aspect of modelling is the incorporation of initial and boundary conditions. Hybrid models necessarily combine the conditions from the two different approaches. For the continuous variables one usually has an initial value $\mathbf{c}_{0}(\mathbf{x})$, while for the agents an initial distribution of their position and internal states is given, which is then used to generate each agents' position at the beginning of the simulation. In some applications the agents can be born during the course of the simulation. In this case, we have to ensure the appropriate initialisation of its internal variables.

A similar idea of independent conditions for the continuum and the agent-based parts of the hybrid model is used for the boundary conditions. The values of the continuous variables on the boundary usually have to satisfy an equation of the type

$$
\mathscr{G}(\mathbf{c}, \mathbf{x}, t)=0, \quad \mathbf{x} \in \partial \Omega,
$$

where $\mathscr{G}$ is a general operator. In the most commonly used cases (12) enforces certain values on $\mathbf{c}$ or its gradient on the boundary. For the agents the boundary conditions are often given in a more descriptive manner. For example, agents can leave the domain through one end and automatically reappear on the other end. This periodic boundary condition implies that the number of agents in the system is conserved. Periodic boundaries are widely used because of their simplicity and because they effectively shape an infinite domain. Reactive boundaries absorb agents with a probability $p$, while reflecting them with probability $1-p$ [14]. If $p=0$, one often speaks of a reflecting boundary, while for $p=1$ the condition is called an absorbing boundary.

\section{Hybrid modelling - numerical implementation}

For similar reasons as in purely agent-based models it is often very hard to obtain analytic results for hybrid models. This increases the importance of numerical simulations for gaining insight into the behaviour of the system. The mixture of different modelling frameworks, however, renders the process of setting up a numerical simulation non-trivial. Each part of the model has to be considered differently and a way of matching the two parts has to be developed. In this section we discuss a numerical framework and evaluate difficulties one has to overcome when implementing a hybrid model.

The general task for the numerical simulation of a hybrid model is to calculate approximations for both $\mathbf{c}$ and $\mathbf{y}_{i}$ at times $t_{j}=j \Delta t, j=1,2, \ldots$ given initial data for each of these variables according to Section 3.2. We additionally assume that the domain $\Omega$ can (for the continuous part of the hybrid model) be adequately represented by the points $\mathbf{r}_{1}, \ldots, \mathbf{r}_{L} \in \Omega$, which means that we seek to compute approximate values for $\mathbf{c}\left(t_{j}, \mathbf{r}_{l}\right), j=1,2, \ldots, l=1, \ldots, L$ and $\mathbf{y}_{i}\left(t_{j}\right), i=1, \ldots, N$. In order to simplify the notation, we introduce 


$$
\underline{C}_{j}=\left[\mathbf{c}\left(t_{j}, \mathbf{r}_{1}\right), \ldots, \mathbf{c}\left(t_{j}, \mathbf{r}_{L}\right)\right] \quad j=0,1, \ldots
$$

Due to the different characters of the continuous and the agent-based subsystems, different approaches have to be used for their numerical solutions. For each of the subsystems one tries to answer the question of how to get from $t_{j}$ to $t_{j+1}$ still guaranteeing an accurate approximation of the system. For the continuous variables this means, we seek a solver that generates the values of $\underline{C}_{j+1}$ using the values $\underline{C}_{0}, \ldots, \underline{C}_{j}$ and the current distribution of the agents $\rho_{\delta}\left(\cdot, t_{j}\right)$ given by (10), which can be symbolised as

$$
\left\{\underline{C}_{0}, \ldots, \underline{C}_{j}, \rho_{\delta}\left(\cdot, t_{j}\right)\right\} \stackrel{\mathscr{L}_{d}}{\longmapsto} \underline{C}_{j+1} .
$$

In (13) we introduced the operator $\mathscr{L}_{d}$, which is a discretised version of the continuous operator $\mathscr{L}$ used in (8). In the most common case, where $\mathscr{L}$ is a differential operator, $\mathscr{L}_{d}$ could be a finite element or finite difference approximation of $\mathscr{L}$. Note that in (13) we have made the implicit assumption that the solver used for (8) only takes the positions of the agents at time $t_{j}$ into account. For the agents equation (11) is already given in a time-discrete way and can therefore be used directly to update the internal states.

The introduction of this general scheme raises some immediate problems, which we will discuss in the remainder of this section. The first difficulty are the differing spatial resolutions for the two subsystems, which we address in Section 4.1. Other problems like time stepping, choices of solvers and the influence of stochastic effects are presented in Section 4.2.

\subsection{Spatial matching in numerical simulations}

A spatial matching between the continuous variables and the agents is required during a numerical simulation of a hybrid system, because different spatial resolutions are applied. The agents can be positioned at an arbitrary point inside the domain $\Omega$, while the data for $\mathbf{c}$ is only calculated at the points $\mathbf{r}_{l}$. This triggers a two-way matching problem, as one has to generate estimates for the agent distribution at the points $\mathbf{r}_{l}$ as well as for the continuous variables $\mathbf{c}$ everywhere inside $\Omega$.

First, let us consider estimating the agent density distribution throughout $\Omega$ and especially at the points $\mathbf{r}_{l}$, which is necessary for the update relation (13). So the general mapping we are trying to achieve is

$$
\rho_{\delta}(\mathbf{x})=\sum_{i=1}^{N} \delta\left(\mathbf{x}-\mathbf{x}_{\mathbf{i}}\right) \stackrel{\Psi}{\longmapsto} \rho(\mathbf{x}) \in C^{0}(\Omega) .
$$

The requirements for the estimated density function $\rho(\mathbf{x})$ can alter for different applications, but here we require it to be at least a continuous function. One way to achieve such a mapping is the so-called kernel density estimation [46]. In general the kernel density estimation can be used to estimate the probability density function of a random process, if one has been given a number of realisations of this process. 
The name stems from the use of a kernel $K(\mathbf{x})$, which is typically a continuous, symmetric and normalised function. Let us for simplicity assume a one dimensional random process, in which case these conditions take the form

$$
K(x) \in C^{0}(\mathbb{R}), \quad K(-x)=K(x), \quad \int_{\mathbb{R}} K(x) \mathrm{d} x=1 .
$$

Additionally, $K(x)$ is often required to be non-negative in order to generate a nonnegative estimate. Most commonly used kernels include a Gaussian kernel and a piecewise linear kernel with compact support. In practice a scaled version of $K$ is used, which leads to the introduction of a bandwidth parameter $h$. We define

$$
K_{h}(x)=\frac{1}{h} K\left(\frac{x}{h}\right)
$$

which still satisfies the conditions (14). With given positions $x_{1}, \ldots, x_{N}$, an estimate of the probability density function is then given by

$$
\rho(x)=\sum_{i=1}^{N} K_{h}\left(x-x_{i}\right)=K_{h}(x) * \rho_{\delta}(x) .
$$

Figure 2 shows an example of a kernel density estimation for 100 normally distributed random variables using a Gaussian kernel with different bandwidths $h$. In Figure 2(a) we can see that the choice of a very small $h$ leads to a highly oscillating estimate, while a very big $h$ can lead to the estimate being too wide as shown in Figure 2(c). An optimal choice for the parameter $h$ and the kernel itself always depends on the nature of the problem and the number of samples $N$.

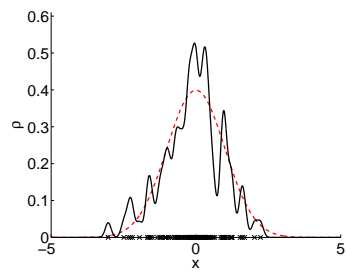

(a) $h=0.1$

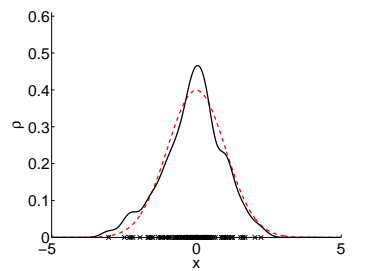

(b) $h=0.25$

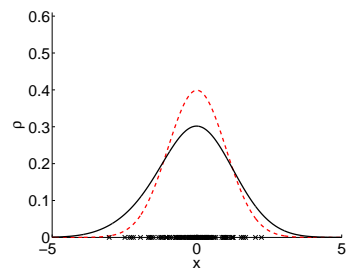

(c) $h=0.9$

Fig. 2 Kernel density estimate for $N=100$ agents, which are placed according to a normal distribution with different bandwidths $h$. Crosses along the x-axis represent the agents, the dashed line is the underlying Gaussian probability density function and the solid line is the generated estimate by (15).

The second spatial matching problem that occurs when simulating a combined continuous and agent-based system is the need to estimate the values of the continuous variables (and possibly their derivatives) at an arbitrary position inside $\Omega$. The operator we are looking for can be symbolised through 


$$
\left(\mathbf{r}_{1}, \mathbf{c}\left(\mathbf{r}_{1}\right)\right), \ldots,\left(\mathbf{r}_{L}, \mathbf{c}\left(\mathbf{r}_{L}\right)\right) \stackrel{\Theta}{\longmapsto} \hat{\mathbf{c}}(\mathbf{x}) \in C^{0}(\Omega) .
$$

Though similar to the operator $\Psi$, we here have the advantage that we know the positions of the points $\mathbf{r}_{1}, \ldots, \mathbf{r}_{L}$ beforehand and that we know they give an adequate representation of the domain $\Omega$. With this additional information, one can argue that the problem at hand represents an interpolation problem from the grid points $\mathbf{r}_{l}$ onto the whole domain $\Omega$. This result allows for the use of approaches from the wellstudied fields of interpolation and approximation theory [47]. In some cases the interpolation regime is already implicitly incorporated in the numerical solution of the update equation for the continuous variables, for example if one chooses to use a finite element approach.

\section{Example 6. Numerical realisation of Example 5}

In Example 5 we presented a hybrid model for chemotaxis of slime mold Dictyostelium discoideum developed by Dallon and Othmer [12]. To generate a discretised operator $\mathscr{L}_{d}$ they used the particle-in-cell method [38]. For the kernel density estimation $\Psi$ they use a piecewise linear kernel and for the interpolation operator $\Theta$ a fifth order spline interpolation was employed.

\subsection{Other aspects of numerical simulations}

The spatial matching between the two parts is the biggest additional challenge posed by the use of a hybrid model. Here, we discuss some other problems that occur during this process. The first problem is the choice of a solver both for the continuous variables and for the internal states of the agents. One can choose from a wide range of standard approaches for both problems. The way the two parts are interwoven, however, sets some restrictions. It is, for example, almost always impossible to use a fully implicit solver for both parts, especially if the functions $\mathbf{f}_{i}$ for the internal agent states contain random variables. Additionally, one has to consider the accuracy of the different solvers and should ideally try to match these to prevent unnecessary computational effort that does not lead to more accurate results.

The discrete nature of the agent-based parts automatically introduces stochastic effects into the system. Various examples of these effects will be discussed in Section 5. It is important to consider these effects when choosing the time stepping and the spatial resolution for the simulation. In particular, these choices will depend on the number of agents in the system. It is generally possible to allow different time steps for different parts of the system, for example the agents could be simulated with a finer time stepping than the continuous variables or vice-versa. For each part of the system the time steps have to be chosen in a way that ensures an accurate solution depending on the spatial resolution and the solver that is used. In Section 5 we study one application area of hybrid systems in more detail and analyse the effect of some of these choices on the system. 


\section{Case study: Hybrid modelling of chemotaxis}

In Section 3 we introduced a general framework for hybrid models that combine agent-based models with mean-field equations and we now concentrate on one application area for hybrid modelling: cell migration. In particular we focus on the movement of cells induced by gradients in the concentration of extracellular chemicals, a process that is known as chemotaxis. Chemotaxis is one of the main forms of cell migration and is used in a variety of cells, including bacteria cells [7]. Hybrid models of chemotaxis have been successfully used in the literature [12, 13, 22, 51].

The first notion of chemotaxis goes back to the late 19th century, when Engelmann and Pfeffer detected the process. In the late 1960s it was Adler [1, 2] who performed experiments with the bacteria $E$. coli that helped understanding and quantifying the process and was later used as comparison for the early mathematical models. Adler placed a colony of E. coli at one end of a long thin pipe that was filled with oxygen and an additional energy source. Through the process of chemotaxis the colony started to move with a constant speed away from the closed end forming a narrow band of bacteria. The band was visible to the naked eye and Adler was able to measure the speed with which it moved forward.

In the 1970s the first mathematical descriptions of chemotaxis were formulated, with the Keller-Segel model, which we will discuss in Section 5.1, as one of the early breakthroughs. A review of the impact this first model had on the modelling of chemotaxis is given in [28]. Section 5.2 will introduce a hybrid version of this model, which we will further investigate and analyse in Section 5.3.

\subsection{The Keller-Segel model}

As mentioned above, Keller and Segel developed the first mathematical model to describe the process of chemotaxis in 1971 [31]. The original model considers both the bacteria and the chemotactic substrate in a continuum limit, which therefore results in a coupled system of two PDEs. The original form of the system only considers one spatial dimension and gives a way to compute the concentration of bacteria denoted by $n(x, t)$ and the concentration of substrate $S(x, t)$ through the PDEs (2)-(3), introduced in Example 1. In equation (2) we can see that the behaviour of the bacteria is governed by two independent effects and therefore takes the form of a general advection-diffusion equation. The diffusion of the bacteria occurs with the diffusion constant $D_{n}$, while the advection is governed by the chemotactic sensitivity $\chi(S)$. The substrate, as seen in (3), diffuses with the diffusion constant $D_{S}$ and is consumed by the bacteria with a consumption rate $k(S)$ that depends on the concentration of substrate itself.

In a follow-up to the paper [31], Keller and Segel showed that under certain conditions the developed system of partial differential equations yields travelling wave solutions [32]. In particular they were able to proof that travelling wave solutions can only exist if $\chi(S)$ has a singularity at some critical value $S_{\text {crit }}$. For reasons 
of simplicity they concentrated on the simplest such functions $\chi(S)=\frac{\kappa}{S}$ with the critical concentration at $S_{\text {crit }}=0$. In their analysis Keller and Segel made some additional assumptions for the various parameter values and simplified (2)-(3) to the nondimensionalised PDEs

$$
\begin{aligned}
& \frac{\partial n}{\partial t}=\mu \frac{\partial}{\partial x}\left(\frac{\partial n}{\partial x}-n \frac{\kappa}{S} \frac{\partial S}{\partial x}\right), \\
& \frac{\partial S}{\partial t}=-n
\end{aligned}
$$

The nondimensionalised system is set up for $x \in[0,1]$ with an initial value of $S(x, 0)=1$ and no-flow boundary conditions. As initial distribution of the agents we choose $n(x, 0)=\delta(x)$, which corresponds to the initial state of Adler's experiments where all bacteria were inserted at one end of the tube. We consider reflective boundary conditions for both bacteria and extracellular signal at $x=0$ and $x=1$.

In order to investigate the influence of the two dimensionless parameters $\mu$ and $\kappa$ on the travelling wave, Figures 3-4 show the concentration of $n$ and $S$ at $t=0.5$ for various values of $\mu$ and $\kappa$. In Figure 3 we can see that the parameter $\mu$ influences the width of the wave while leaving its general shape untouched. Increasing $\mu$ leads to a wider wave and a decrease in the maximum of $n$. Accordingly, the gradient in $S$ is higher for the narrower bands caused by smaller values of $\mu$. As can be seen in Figure 4 , the parameter $\kappa$ influences the general shape of the wave. In the case $\kappa=2$ the travelling band of bacteria is symmetric, while a $\kappa$ bigger than two leads to a wave that is steeper in the front (right) and falls slowly in the back (left) of the wave. Choosing $\kappa$ smaller than two causes an opposite effect with the wave being bent backwards.

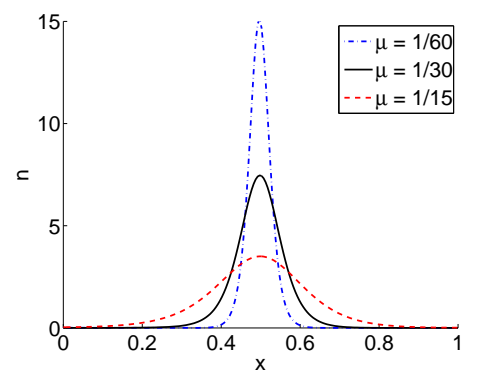

(a) $n(x, 0.5)$

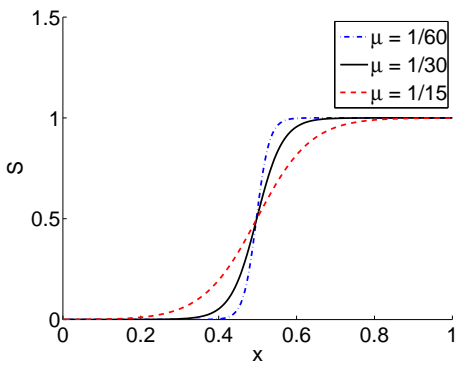

(b) $S(x, 0.5)$

Fig. 3 Travelling wave solution of the Keller-Segel model (16)-(17) for different values of the parameter $\mu$, where $\kappa=2$. 


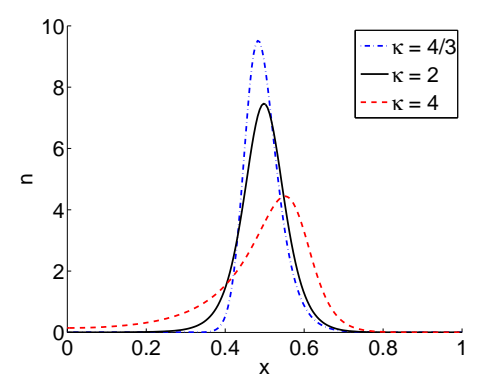

(a) $n(x, 0.5)$

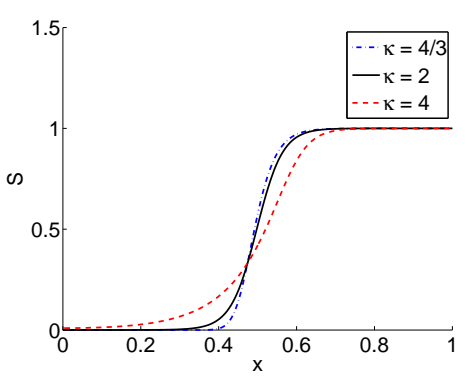

(b) $S(x, 0.5)$

Fig. 4 Travelling wave solution of the Keller-Segel model for different values of the parameter $\kappa$, where $\mu=1 / 30$.

\subsection{Hybrid models of chemotaxis}

One of the assumptions made by Keller and Segel in their original model is to consider the bacteria as a continuum rather than explicitly describe their individual behaviour. For systems that do not satisfy this assumption hybrid chemotaxis models have been developed in the literature $[12,13,22,51]$. In this section we present three of them. The bacteria are modelled as agents with varying numbers of internal states and their position $x_{i} \in \Omega$, as the only observable state. All three models consider the substrate in a continuum limit and the PDE (17) takes the role of equation (8) in our description of the hybrid modelling framework.

\section{Model I}

The first approach to design a hybrid version of the Keller-Segel model, is to interpret the evolution equation for $n$ as a Fokker-Planck equation for a number of randomly moving particles similarly to the idea presented in Example 3. A chemotaxis model of this form was formulated by Stevens [43]. The movement of each of the agents is described by the stochastic differential equation

$$
\mathrm{d} x_{i}=\mu \frac{\kappa}{S\left(x_{i}\right)} \frac{\partial S\left(x_{i}\right)}{\partial x} \mathrm{~d} t+\sqrt{2 \mu} \mathrm{d} W
$$

The parameters used in (18) correspond to the ones in the dimensionless KellerSegel equations (16)-(17). This particle-based description of equation (16) shows one of the weaknesses in the original Keller-Segel model. According to (18) an agent can theoretically jump any given distance in one time step, implying that some of them can move with a speed that is not achievable for bacteria. 


\section{Model II}

Driven by weaknesses of the first model, a different type of random walk, known as velocity-jump process, seems a more realistic choice for the bacterial behaviour. The motion of bacteria E. coli consists of two phases [7]. During a run-phase the bacterium moves with a constant speed straight into a chosen direction. This run lasts for a randomly distributed time before the bacterium enters the tumble-phase in which it chooses a new direction randomly [6]. As we are considering a onedimensional model, there are only two possible directions of motion: to the left and to the right. A right-moving agent continues to the right for a time that is given by an exponentially distributed random variable before it switches its direction. In order to incorporate the bias of bacteria towards higher concentrations of chemoattractants, Othmer et al. [37] introduced a biased velocity-jump process. In this biased random walk the duration for the run phase depends on information gathered at the current position of the individual. In particular, the model in [37] allows the agents to directly measure the gradient of the substrate concentration at their current position. The run-phase then tends to be longer, if the concentration increases in the current direction of motion, while for a decreasing signal, the turning probability is increased.

The turning frequency $\lambda$ is therefore adjusted according to the current movement direction, the value and the gradient of $S$. To represent the direction of motion, the velocity $v_{i}(t)= \pm s$ is introduced, where $s$ denotes the constant speed. In terms of the hybrid modelling framework introduced in Section 3, the internal variable is $\mathbf{y}_{i}=\left[x_{i}, v_{i}\right]$. The agent-based description of the bacteria can be written in the form

$$
\begin{aligned}
& x_{i}(t+\Delta t)=x_{i}(t)+v_{i}(t) \mathrm{d} t, \\
& v_{i}(t+\Delta t)=\left\{\begin{array}{rl}
-v_{i}(t) & \text { with probability } \lambda^{ \pm} \Delta t \\
v_{i}(t) & \text { otherwise }
\end{array},\right.
\end{aligned}
$$

where

$$
\lambda^{ \pm}=\lambda_{0} \mp \frac{\kappa s}{2 S} \frac{\partial S}{\partial x}
$$

In a continuum limit this velocity-jump process is equivalent to the hyperbolic chemotaxis equation [16]:

$$
\frac{1}{2 \lambda_{0}} \frac{\partial^{2} n}{\partial t}+\frac{\partial n}{\partial t}=\frac{s^{2}}{2 \lambda_{0}} \frac{\partial}{\partial x}\left(\frac{\partial n}{\partial x}-n \frac{\kappa}{S} \frac{\partial S}{\partial x}\right),
$$

where $n$ is the concentration of bacteria. This shows that changing the type of random-walk used for the agents can influence the corresponding continuum equation. Nevertheless (19) can be used to adjust the parameters of the agent-based model to match the parameters of the Keller-Segel model, as the large time behaviour of (19) is given by the classical chemotaxis equation (16), where we have $\mu=s^{2} /\left(2 \lambda_{0}\right)$ [30]. Lui et al. [34] showed that coupling the hyperbolic chemotaxis equation (19) with (3) for the substrate also yields travelling wave solutions similar 
to the original Keller-Segel system. An investigation of this case for a more general dependence of the turning frequency is given in [50].

\section{Model III}

More accurate descriptions of the individual behaviour of bacteria incorporate the sensing and processing of extracellular signals [5, 42]. Hybrid models with descriptions of these intracellular processes have been used by Dallon and Othmer [12] as well as Xue et al. [49]. Erban and Othmer [17, 18] used an agent with a toy version of the internal dynamics that includes two main features of the sensing process: a fast excitation and a slower adaptation. We will use a simple model with one additional internal variable $z_{i}$ that acts as a memory and allows the agent to identify increasing or decreasing signal concentrations [17]. The model is based on a velocity-jump process with a turning frequency $\lambda$, which depends on $z_{i}$. This internal variable is chosen to follow the value of a sensing function $g(S)$ with the adaptation time $t_{a}$. Thus, the model can be written in the hybrid form presented in Section 3, using $\mathbf{y}_{i}=\left[x_{i}, v_{i}, z_{i}\right]$ as follows:

$$
\begin{aligned}
& x_{i}(t+\Delta t)=x_{i}(t)+v_{i}(t) \mathrm{d} t, \\
& v_{i}(t+\Delta t)=\left\{\begin{array}{r}
-v_{i}(t) \text { with probability } \lambda \Delta t, \\
v_{i}(t) \text { otherwise },
\end{array}\right. \\
& z_{i}(t+\Delta t)=z_{i}(t)+\frac{g\left(S\left(x_{i}(t)\right)\right)-z_{i}(t)}{t_{a}} \Delta t,
\end{aligned}
$$

where

$$
\lambda=\lambda_{0}+z_{i}-S\left(x_{i}\right) .
$$

In the limit $\Delta t \rightarrow 0$ and $N \rightarrow \infty$ this process can be described by the chemotaxis equation

$$
\frac{\partial n}{\partial t}=\frac{s^{2}}{2 \lambda_{0}} \frac{\partial}{\partial x}\left(\frac{\partial n}{\partial x}-\frac{2 t_{a}}{1+2 \lambda_{0} t_{a}} \frac{\mathrm{d} g}{\mathrm{~d} S} \frac{\partial S}{\partial x}\right),
$$

provided that $t$ is large $\left(t \gg 1 / \lambda_{0}\right)$ and the gradient of $S$ is shallow [17]. Choices for the parameters of this model can be made by matching (20) with the classical chemotaxis equation (16), which especially indicates that $g$ is given through $\mathrm{d} g / \mathrm{d} S \sim \chi(S)$.

In Figure 5(a) a simulation of the hybrid model of type III is shown. Simulations of the other two models were also performed, with results almost identical to the one seen in Figure 5(a). We simulate $N=10^{4}$ agents with the dimensionless model parameters $1 / t_{a}=\lambda_{0}=1.5 \times 10^{-3}, s=10^{-2}, g(S)=4.5 \times 10^{-3} \log (S)$ and $\Delta t=10^{-4}$. These parameters were chosen in such a way that they match the global parameters $\mu=1 / 30$ and $\kappa=2$ used for the classical Keller-Segel model. On a first impression, it looks as though the resulting agent distribution at $t=0.5$ matches the predicted concentration of the Keller-Segel system well except for some stochastic effects. In Figure 5(b), however, we show the agent distribution in the region behind 
the travelling band. Further analysis of this region showed that here the extracellular signal is completely exploited. Some agents are left in this zone and undergo an unbiased random walk without a chemotactic signal to guide them. This means that these agents do not necessarily manage to catch up with the travelling wave again but instead stay in the exploited region. In the remainder of this section, we study this effect, which we refer to as dropout in more detail. We will show that it significantly influences the system dynamics for large times.

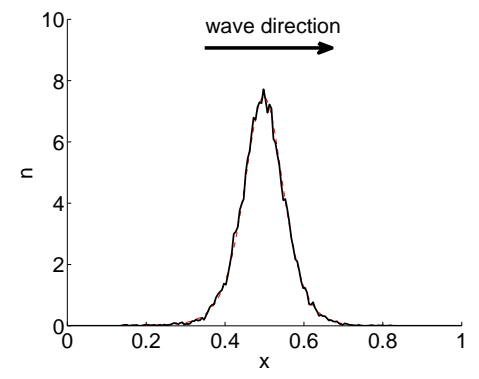

(a)

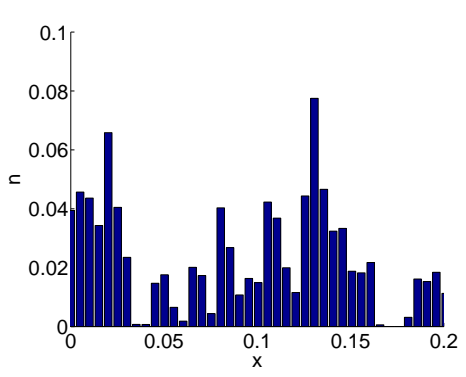

(b)

Fig. 5 Numerical Simulation of the hybrid Keller-Segel model with internal dynamics (Model III). Parameters are $N=10^{4}, 1 / t_{a}=\lambda_{0}=1.5 \times 10^{-3}, s=10^{-2}, g(S)=4.5 \times 10^{-3} \log (S), \Delta t=10^{-4}$. (a) Distribution of agents at time 0.5 (solid line) and the results given by the Keller-Segel model (16)-(17) (dashed line, which is almost indistinguishable from the solid line).

(b) Histogram of agent positions in subinterval $[0,0.2]$.

\subsection{Analysis of the dropout}

In Figure 5(b) we saw that the hybrid model, in contrast to the original Keller-Segel model, creates a region behind the wave where the substrate is completely exploited. The main assumptions for a mean-field approach are violated in this region, namely the number of bacteria and the concentration of extracellular material are very small, which renders a continuum approach here not applicable. Stochastic effects due to the small number of bacteria then lead to the complete exploitation of $S$, which causes the dropout of some of the agents. These agents can no longer sense any gradient in extracellular substrate and are therefore moving completely randomly, which makes it very unlikely for them to become part of the travelling band again. Due to the constant loss of agents, the velocity and the height of the wave will decrease as the wave moves along. Note that a complete exploitation in these models is only possible under the assumption that $S$ does not diffuse, which was made by Keller and Segel and is incorporated in the PDE (17). The dropout effect is interesting for us, because it shows a qualitative difference between the hybrid model and the original Keller-Segel model, as the hybrid model only yields transient travelling 
wave solutions. In this section we create measures for this dropout in order to get an estimate of the number of lost agents from the simulations. We will then move on to analyse the effect of some system parameters on the dropout. Finally, some theoretical results about the loss of agents are presented and compared to numerical results.

\section{Dropout measures}

In order to be able to quantify the dropout of agents from the travelling wave, we need to investigate certain conditions that render an agent as dropped out. A condition of this form allows us to define an index set $\Gamma(t)$ that contains the agents who are currently part of the wave.

However, before defining and comparing different conditions for the dropout, we investigate some global statistical values of the agent set. The first measure to indicate the fact that agents have dropped out is the position of the centre of the wave $c(t)$. From [32] we know that the theoretical wave speed of the nondimensionalised Keller-Segel system is 1 and therefore the predicted position of the centre of the wave is $c_{m f}(t)=t$. In comparison to that the actual position of the wave can be measured from the agents' positions via

$$
c_{1}(t)=\frac{1}{N} \sum_{i=1}^{N} x_{i}(t) .
$$

The problem with this option is that it includes dropped out agents for the calculation of the wave centre, which can bias the calculation. To overcome this problem, a second option for finding the centre of the wave is given through

$$
c_{2}(t)=\frac{1}{|\Gamma|} \sum_{i \in \Gamma} x_{i}(t)
$$

which implies that the found centre position depends on the choice for the index set $\Gamma$. For short times $c_{1}(t)$ and $c_{2}(t)$ give similar results, but will differ for large times. Using this wave centre $c_{1}(t)$, we can calculate the variance of the agent positions as an indicator for the width of the wave and therefore for the dropout. In Figure 6(a) this variance is compared to the variance of the travelling wave solution found by Keller and Segel, which is $\sigma_{m f}=(\pi \mu)^{2} / 3$. Initially the measured variance increases linearly towards the theoretical value, which is caused by the start of the agents on the boundary $x=0$. After the wave is fully developed, the variance starts to rise over the theoretical value, which indicates a significantly wider wave and therefore dropout of agents.

With these statistical values for the agent set we have now different options to define an agent as dropped out from the wave and therefore to define the index set $\Gamma$. The first option is to allow an agent to have a certain distance $r$ from the centre of the wave. Agents with a distance bigger than $r$ are therefore considered to be 
dropped out. Hence,

$$
\Gamma_{1} \equiv \Gamma_{1}(t ; r)=\left\{i \in\{1, \ldots, N\} \mid x_{i}(t) \geq c_{1}(t)-r\right\} .
$$

Because of the non-finite support of the travelling wave solution for the original Keller-Segel system, the measure defined in (23) is strongly dependent on $r$, which makes the choice of $r$ important. One should choose $r$ in a way that the solution of the original Keller-Segel model only predicts a very small number of dropout agents. One way to pick $r$ is to use a multiple of the theoretical standard deviation of the wave.

A second option of defining an agent as dropped out is to use the observation that $S$ is exploited behind the wave. An agent is then considered to be dropped out of the wave if the value of $S$ at its current position is 0 . Thus,

$$
\Gamma_{2} \equiv \Gamma_{2}(t)=\left\{i \in\{1, \ldots, N\} \mid S\left(x_{i}(t)\right)=0\right\} .
$$

Using the sets $\Gamma_{1}$ and $\Gamma_{2}$ we can now define 2 dropout measures $d_{1}(t ; r)$ and $d_{2}(t)$ by

$$
d_{1}(t ; r)=1-\frac{1}{N}\left|\Gamma_{1}(t ; r)\right|, \quad \text { and } \quad d_{2}(t)=1-\frac{1}{N}\left|\Gamma_{2}(t)\right| .
$$

Figure 6(b) shows plots of the behaviour of $d_{1}(t ; r)$ and $d_{2}(t)$. We can see that after the initial period of adjustment due to the start on the boundary $x=0$, all measures have an increasing trend with some fluctuations around it. The measure $d_{1}(t ; 0.15)$ matches well with $d_{2}(t)$, but has less fluctuations.

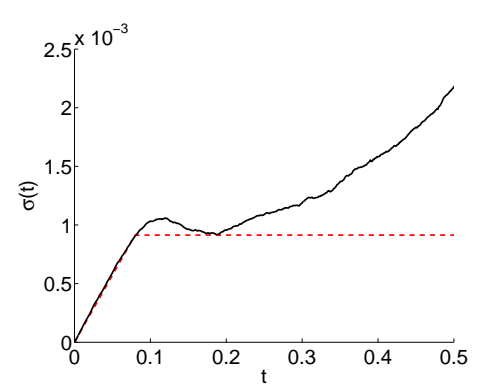

(a)

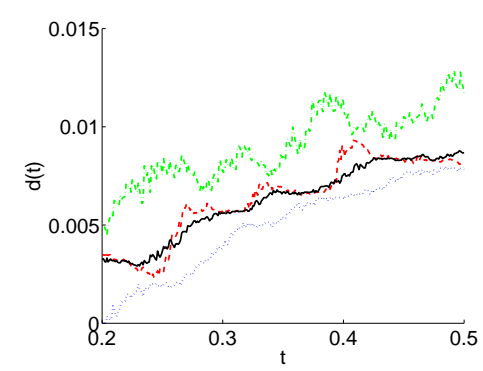

(b)

Fig. 6 Simulation results of the variance and dropout for short times, where the same parameter values as in Figure 5 are used.

(a) Variance $\sigma(t)$ estimated from the simulation (solid line) and variance of the stationary wave given by the mean-field model $\sigma_{m f}$ (dashed line).

(b) Dropout given by (25): $d_{1}(t ; 0.1)$ (dash-dotted line), $d_{1}(t ; 0.15)$ (solid line), $d_{1}(t ; 0.2)$ (dotted line) and $d_{2}(t)$ (dashed line). 


\section{Large time behaviour}

In this section we investigate the large time behaviour of the travelling wave in the hybrid chemotaxis Model III. We study the behaviour of the bacteria and the signal in the half-line $[0, \infty]$. For large times the definitions $c_{1}(t)$ and $c_{2}(t)$ given by (21)(22) differ significantly because many agents drop behind the wave. Therefore, $c_{2}(t)$ is more meaningful to describe the centre of the wave in this case. However, as $c_{2}(t)$ depends on $\Gamma$, we can no longer use $\Gamma \equiv \Gamma_{1}$ to find the agents that have dropped out, because $\Gamma_{1}$ depends on the definition of the centre of the wave. We therefore use $d_{2}(t)$ given by (25) as measure for the dropout in the analysis of large time behaviour, where we are particularly interested in the slowing down of the wave. Hence, we define the velocity of the wave $v(t)$ through

$$
v(t)=\frac{c_{2}(t+\Delta T)-c_{2}(t)}{\Delta T},
$$

where $\Delta T$ is chosen to be much larger than $\Delta t$ in order to minimise the fluctuations in $v(t)$. We simulate $N=10^{4}$ agents with the same parameters as before. The results of one simulation are shown in Figure 7 . We see that after $t=50$ about $40 \%$ of the agents have dropped out from the wave. The predicted slowing down of the wave is demonstrated in Figure 7(b), where we plot $v(t)$ as a function of time. We use $\Delta T=0.1$ in the definition (26). As the velocity shrinks with the number of agents in the wave, we have $v(t) \approx 1-d_{2}(t)$, which is also demonstrated in Figure 7(b).

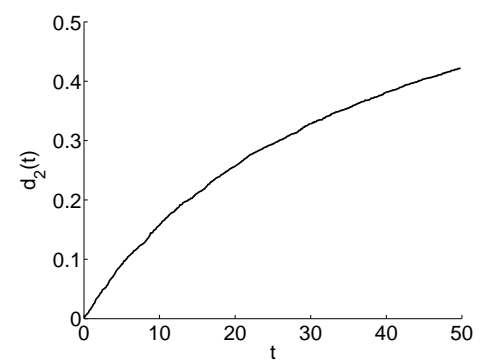

(a)

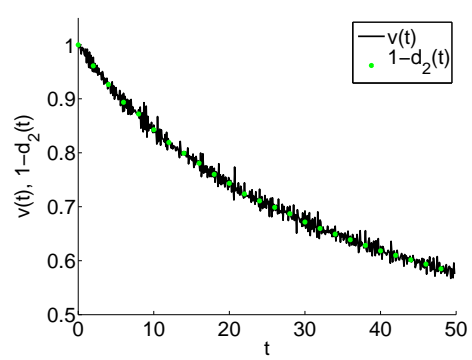

(b)

Fig. 7 Dropout and velocity of the travelling wave for large time, where the same parameter values as in Figure 5 are used.

(a) Dropout $d_{2}(t)$ given by (25).

(b) Velocity of the wave $v(t)$ given by (26) (solid line) compared with $1-d_{2}(t)$ (circles).

\section{Dropout in dependence on $N$ and $\Delta x$}

In the next step we use the derived measure (25) in order to analyse the influence of certain system parameters on the dropout. In particular, we are interested in the 
dependence of the dropout on the number of agents $N$ and the gridsize $\Delta x$. The variation of the number of agents $N$ in the system is a way of comparing the hybrid with the continuum model. One would expect that the dropout goes to 0 as $N$ goes to infinity. On the other hand the $\Delta x$ dependence is a problem of the hybrid model, as one would ideally want the dropout to be independent of the chosen grid. We performed a number of simulations for various values of $N$ (200 simulations for each value) and $\Delta x$ (100 simulations for each value) and in each case measured the value of $d_{1}(0.5 ; 0.15)$. The results are plotted in Figure 8. In Figure 8(a) we plot

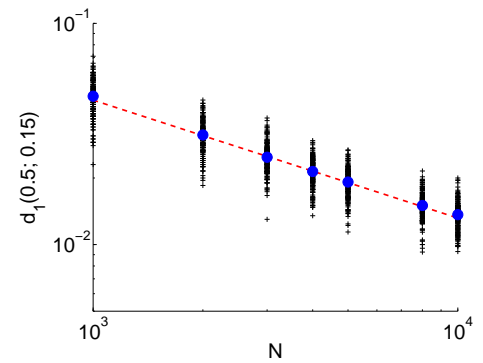

(a)

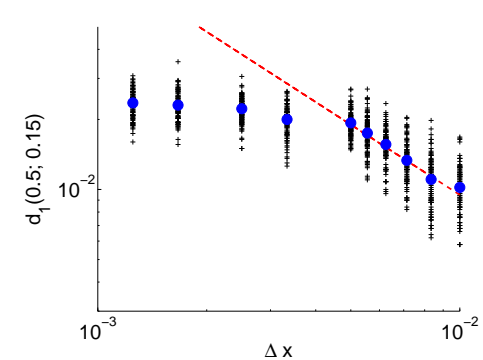

(b)

Fig. 8 Dropout $d_{1}(0.5 ; 0.15)$ given by (25) as a function of (a) $N$ and (b) $\Delta x$. In each figure we show results given by individual simulations (crosses), average values of $d_{1}(0.5 ; 0.15)$ estimated from simulations (circles) and linear fits explained in the text (dashed-line).

the average values of $d_{1}(0.5 ; 0.15)$ estimated from simulations as circles. The best linear fit in the double logarithmic plot, shown as the dashed line, has a gradient of -0.53 , which indicates that $d_{1} \sim 1 / \sqrt{N}$. This relationship can be explained through the central limit theorem, which predicts that the noise in the system should shrink with $\sqrt{N}$.

The plot in Figure 8(b) shows a more complicated dependence. For larger values of $\Delta x$ the dashed line with gradient -1 can be fitted indicating that a finer grid leads to an increase in dropout, which seems slightly surprising at first glance, as one expects a finer grid to allow for a more accurate representation of the original PDE. This effect can, however, be explained by the lower number of agents per gridpoint and therefore the higher noise expected at each gridpoint. As $\Delta x$ decreases the dropout seems to level off, meaning that the choice of a finer grid at this point does not influence the dropout drastically. Bearing in mind that we ideally wanted the dropout to be independent of $\Delta x$, this levelling off effect seems to indicate the region of choice for $\Delta x$ in order to get an accurate solution.

\section{Theoretical analysis}

More theoretical insight into the dropout effect can be obtained by considering a simplified system, where the concentration of extracellular material $S$ is a given 
function that does not change over time. A natural choice for the function $S(x)$ is the travelling wave solution found by Keller and Segel [32]. Using the knowledge of the exploited region behind the wave, we can adjust this function slightly to allow for the analysis of the dropout effect. We therefore define $S$ to be equal to 0 for $x$ smaller than some critical position $x_{c}$ and to take the form of the travelling wave solution everywhere else. In this section we will use $\kappa=2$, thus we put

$$
S(x)= \begin{cases}(1+\exp (-x / \mu))^{-1}, & x \geq x_{c} \\ 0, & x<x_{c}\end{cases}
$$

To be able to use a time-independent function for $S$ we have to make adjustments to the movement of the agents, as they would otherwise follow the increasing gradient towards the right of the real axis. Therefore, we subtract the expected wave speed of 1 from the movement velocity of the agents in order to keep them in a position that is realistic for the travelling wave. In other words, we use a coordinate system that moves with the travelling wave solution. For example, for an agent of Model I the evolution equation becomes

$$
\mathrm{d} x_{i}=\left(\mu \frac{2}{S\left(x_{i}\right)} \frac{\mathrm{d} S\left(x_{i}\right)}{\mathrm{d} x}-1\right) \mathrm{d} t+\sqrt{2 \mu} \mathrm{d} W .
$$

With the help of this simplified system we can now make further analytic and simulative investigations into the effect of different $x_{c}$ on the quantity of the dropout. If an agent enters the exploited region $x<x_{c}$, two behaviours are considered. In the first case, the agent would be considered dropped out and is absorbed by the boundary, so that it has no chance of becoming part of the wave again. The second case allows the agent to randomly move around in the exploited area and therefore allows the agent to enter the non-exploited region again. For both cases we performed 100 simulations for each of the considered values of $x_{c}$ and measured the value of $d_{1}(0.5 ; 0.15)$ as defined before, this time using 0 as the mean position. The average values of $d_{1}(0.5 ; 0.15)$ estimated from the simulations are shown in Figure 9 as circles. To analyse the case of an absorbing boundary at $x=x_{c}$ we consider the system in the limit $N \rightarrow \infty$, which is described by the following equation (compare to (16))

$$
\frac{\partial n}{\partial t}-\frac{\partial n}{\partial x}=\mu \frac{\partial}{\partial x}\left(\frac{\partial n}{\partial x}-n \frac{2}{S} \frac{\mathrm{d} S}{\mathrm{~d} x}\right) .
$$

The boundary condition on the left-hand boundary can be written in the form $n\left(x_{c}\right)=0$. Further conditions for $x \rightarrow \infty$ can be introduced. We look for a separable solution of the form

$$
n(x, t)=\exp (-\lambda t) M(x),
$$

where $\lambda$ is a positive constant. Plugging this ansatz into (28) leads to

$$
\mu M^{\prime \prime}+M^{\prime}-2 \mu\left(M \frac{S^{\prime}}{S}\right)^{\prime}+\lambda M=0,
$$




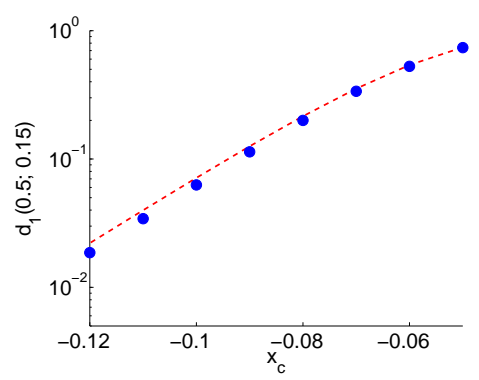

(a)

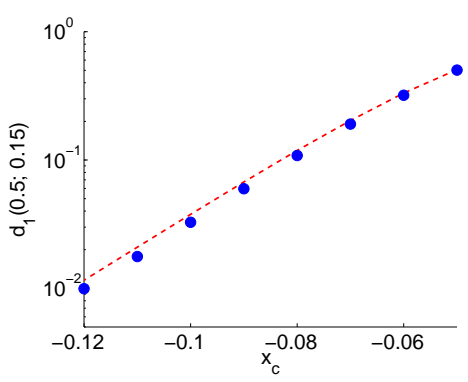

(b)

Fig. 9 Dropout $d_{1}(0.5 ; 0.15)$ defined by (25) as a function of $x_{c}$ for static signal given by (27) where the same parameter values as in Figure 5 are used. In each figure we show average values of $d_{1}(0.5 ; 0.15)$ estimated from 100 simulations (circles).

(a) Simulations where no comeback from $x=x_{c}$ is allowed. The dashed line is a result of the theoretic analysis given by (30).

(b) Simulations where dropout agents can return. The dashed line is $50 \%$ of the dropout predicted by (30).

where primes denote derivatives with respect to $x$. For the ODE (29) a non-negative solution is sought that satisfies $M\left(x_{c}\right)=0$ and $M(x) \rightarrow 0$ as $x \rightarrow \infty$. The general solution for (29) is

$$
\begin{aligned}
M(x) & =C_{1} \frac{2 \lambda \mu \exp \left(x \frac{3+\gamma}{2 \mu}\right)+(1+\gamma+2 \lambda \mu) \exp \left(x \frac{1+\gamma}{2 \mu}\right)}{\left(\exp \left(\frac{x}{\mu}\right)+1\right)^{2}} \\
& -C_{2} \frac{2 \lambda \mu \exp \left(x \frac{3-\gamma}{2 \mu}\right)+(1-\gamma-2 \lambda \mu) \exp \left(x \frac{1-\gamma}{2 \mu}\right)}{\left(\exp \left(\frac{x}{\mu}\right)+1\right)^{2}}
\end{aligned}
$$

where $\gamma=\sqrt{4 \lambda \mu+1}$. The integration constants $C_{1}$ and $C_{2}$ have to be chosen to satisfy the boundary conditions. Because of the nature of (29) as an eigenvalue problem, only the quotient $C_{1} / C_{2}$ can be determined uniquely, which also means that the condition $M(x) \rightarrow 0$ as $x \rightarrow \infty$ is satisfied for all values $C_{1}, C_{2} \in \mathbb{R}$. Taking a closer look at the limit $x \rightarrow \infty$, we can see that the direction of the approach changes in dependence of $\lambda$, in particular, a non-negative solution can only be obtained for $\lambda$ smaller than a critical value $\lambda_{c}\left(x_{c}\right)$. This critical value $\lambda_{c}\left(x_{c}\right)$ is achieved for the case where $C_{1} / C_{2}$ turns out to be 0 . Applying the left-hand boundary condition $M\left(x_{c}\right)=0$ for this case yields to the unique value $\lambda_{c}\left(x_{c}\right)$ given through

$$
\lambda_{c}\left(x_{c}\right)=-\frac{1}{\mu} \exp \left(-\frac{x_{c}}{\mu}\right)\left(1+\exp \left(-\frac{x_{c}}{\mu}\right)\right)^{-2}=-S^{\prime}\left(x_{c}\right) \text {. }
$$


This value $\lambda_{c}\left(x_{c}\right)$ can now be used to get a predicted value of the dropout $d_{\text {pred }}\left(x_{c}, t\right)$ via

$$
d_{\text {pred }}\left(x_{c}, t\right)=1-\exp \left(\lambda\left(x_{c}\right) t\right) .
$$

The function $d_{\text {pred }}\left(x_{c}, 0.5\right)$ is plotted as the dashed line in Figure 9(a). We can see that it matches well with the simulation results. The slight overestimation given by $d_{\text {pred }}\left(x_{c}, 0.5\right)$ can be explained through the time it takes before the first agents start reaching the critical position $x_{c}$ from the starting position at $x=0$. For the situation with comeback, we choose a value $\lambda=\alpha \lambda_{c}\left(x_{c}\right)$ to predict the dropout, where $\alpha$ is a constant. Matching this with the simulation results as shown in Figure 9(b) we found that $\alpha \approx 0.5$, which indicates that about $50 \%$ of agents come back into the wave after they have dropped out. This effect could be modelled by using a reactive boundary [14] instead of the free diffusion zone behind the wave.

\section{Conclusion}

In this chapter we reviewed the advances that have been made in the field of hybrid modelling of collective behaviour. Hybrid models combine agent-based models with mean-field concentration models and allow a more accurate description of certain systems than the general mean-field approach. Compared to purely agent-based models hybrid models have the advantage of a reduced computational complexity and a wider range of applicability. As hybrid models explicitly consider individual behaviour as well as interactions between individuals, stochastic effects are incorporated which can alter the behaviour from that of the corresponding continuum model. This became especially clear during the studies of hybrid chemotaxis models in Section 5. We showed that the hybrid models do not produce a travelling wave in the classical sense, as agents are dropping out behind the wave. This effect leads to a decrease in the number of agents in the wave, which also slows down the wave, as demonstrated in Figure 7. We also discussed some of the problems and difficulties related to the use of hybrid models. In particular the spatial matching between the discrete agents and the continuous variables has to be considered. We showed in Figure 8 that the choice of the gridsize can have a significant effect on the behaviour of hybrid models and has to be handled with care.

\section{Acknowledgements}

The research leading to these results has received funding from the European Research Council under the European Community's Seventh Framework Programme (FP7/2007-2013) / ERC grant agreement No. 239870. This publication was based on work supported in part by Award No KUK-C1-013-04, made by King Abdul- 
lah University of Science and Technology (KAUST). RE would also like to thank Somerville College, University of Oxford, for a Fulford Junior Research Fellowship.

\section{References}

1. Adler, J.: Chemotaxis in Bacteria. Science 153, 708-716 (1966)

2. Adler, J.: Chemoreceptors in Bacteria. Science 166, 1588-1597 (1969)

3. Alarcón, T., Byrne, H., Maini, P.: A cellular automaton model for tumour growth in inhomogeneous environment. Journal of Theoretical Biology 225, 257-274 (2003)

4. Anderson, A.: A hybrid mathematical model of solid tumour invasion: the importance of cell adhesion. Mathematical Medicine and Biology : a Journal of the IMA 22, 163-186 (2005)

5. Barkai, N., Leibler, S.: Bacterial chemotaxis - united we sense ... Nature 393, 18-21 (1998)

6. Berg, H.: How bacteria swim. Scientific American 233, 36-44 (1975)

7. Berg, H., Brown, D.: Chemotaxis in Esterichia coli analysed by three-dimensional tracking. Nature 239, 500-504 (1972)

8. Bobashev, G., Goedecke, M., Yu, F., Epstein, J.: A Hybrid Epidemic Model: Combining the Advantages of Agent-Based and Equation-Based Approaches. In: S. Henderson (ed.) Proceedings of the 2007 Winter Simulation Conference, pp. 1532-1537. IEEE (2007)

9. Buhl, J., Sumpter, D., Couzin, I., Hale, J., Despland, E., Miller, E., Simpson, S.: From disorder to order in marching locusts. Science 312, 1402-1406 (2006)

10. Couzin, I., Krause, J., Franks, N., Levin, S.: Effective leadership and decision-making in animal groups on the move. Nature 433, 513-516 (2005)

11. Couzin, I., Krause, J., James, R., Ruxton, G., Franks, N.: Collective memory and spatial sorting in animal groups. Journal of Theoretical Biology 218(1), 1-11 (2002)

12. Dallon, J., Othmer, H.: A discrete cell model with adaptive signalling for aggregation of dictyostelium discoideum. Philosophical Transactions of the Royal Society B: Biological Sciences 352(1351), 391-417 (1997)

13. Erban, R.: From individual to collective behaviour in biological systems. Ph.D. thesis, University of Minnesota (2005)

14. Erban, R., Chapman, S.J.: Reactive boundary conditions for stochastic simulations of reactiondiffusion processes. Physical Biology 4(1), 16-28 (2007)

15. Erban, R., Chapman, S.J.: Time scale of random sequential adsorption. Physical Review E 75(4), 041,116 (2007)

16. Erban, R., Kevrekidis, I., Othmer, H.: An equation-free computational approach for extracting population-level behavior from individual-based models of biological dispersal. Physica D 215(1), 1-24 (2006)

17. Erban, R., Othmer, H.: From individual to collective behaviour in bacterial chemotaxis. SIAM Journal on Applied Mathematics 65(2), 361-391 (2004)

18. Erban, R., Othmer, H.: From signal transduction to spatial pattern formation in E. coli: A paradigm for multi-scale modeling in biology. Multiscale Modeling and Simulation 3(2), 362-394 (2005)

19. Flegg, M., Chapman, J., Erban, R.: The Two Regime Method for optimizing stochastic reaction-diffusion simulations (2011). Submitted

20. Franz, B.: Synchronisation Properties of an Agent-Based Animal Behaviour Model. Master's thesis, University of Oxford (2009)

21. Gerlee, P., Anderson, A.: An evolutionary hybrid cellular automaton model of solid tumour growth. Journal of Theoretical Biology 246, 583-603 (2007)

22. Guo, Z., Sloot, P., Tay, J.C.: A hybrid agent-based approach for modeling microbiological systems. Journal of Theoretical Biology 255(2), 163-175 (2008)

23. Helbing, D., Farkas, I., Vicsek, T.: Simulating dynamical features of escape panic. Nature 407, 487-490 (2000) 
24. Hellweger, F., Bucci, V.: A bunch of tiny individuals: Individual-based modeling for microbes. Ecological Modelling 220, 8-22 (2009)

25. Heppenstall, A., Evans, A., Birkin, M.: A Hybrid Multi-Agent / Spatial Interaction Model System for Petrol Price Setting. Transactions in GIS 9, 35-51 (2005)

26. Heppenstall, A., Evans, A., Birkin, M.: Using Hybrid Agent-Based Systems to Model Spatially-Influenced Retail Markets. Journal of Artificial Societies and Social Simulation 9, 2 (2006)

27. Heppenstall, A., Evans, A., Birkin, M.: Genetic algorithm optimisation of an agent-based model for simulating a retail market. Environment and Planning B: Planning and Design 34, 1051-1070 (2007)

28. Horstmann, D.: From 1970 until present: the Keller-Segel model in chemotaxis and its consequences i. Jahresbericht der DMV 105(3), 103-165 (2003)

29. van Kampen, N.: Stochastic Processes in Physics and Chemistry, 3rd edn. North-Holland, Amsterdam (2007)

30. Karch, G.: Selfsimilar profiles in large time asymptotics of solutions to damped wave equations. Studia Mathematica 143, 175-197 (2000)

31. Keller, E., Segel, L.: Model for chemotaxis. Journal of Theoretical Biology 30, 225-234 (1971)

32. Keller, E., Segel, L.: Traveling bands of chemotactic bacteria: A theoretical analysis. Journal of Theoretical Biology 30, 235-248 (1971)

33. Landsberg, J., Waring, R.: A generalised model of forest productivity using simplified concepts of radiation-use efficiency, carbon balance and partitioning. Forest Ecology and Management 95, 209-228 (1997)

34. Lui, R., Wang, Z.A.: Traveling wave solutions from microscopic to macroscopic chemotaxis models. Journal of Mathematical Biology 61(5), 739-761 (2010)

35. Murray, J.: Mathematical Biology. Springer-Verlag (2002)

36. Osborne, J., Walter, A., Kershaw, S., Mirams, G., Fletcher, A., Pathmanathan, P., Gavaghan, D., Jensen, O., Maini, P., Byrne, H.: A hybrid approach to multi-scale modelling of cancer. Philosophical Transactions of the Royal Society A: Mathematical, Physical and Engineering Sciences 368, 5013-5028 (2010)

37. Othmer, H., Dunbar, S., Alt, W.: Models of dispersal in biological systems. Journal of Mathematical Biology 26, 263-298 (1988)

38. P., O., Brackbill, J.: On particle-grid interpolation and calculating chemistry in particle-in-cell methods. Journal of Computational Physics 103, 37-52 (1993)

39. Patel, A., Gawlinski, E., Lemieux, S., Gatenby, R.: A cellular automaton model of early tumor growth and invasion. Journal of Theoretical Biology 213, 315-331 (2001)

40. Ribba, B., Alarcón, T., Marron, K., Maini, P., Agur, Z.: The use of hybrid cellular automaton models for improving cancer therapy. In: P. Sloot, B. Chopard, A. Hoekstra (eds.) ACRI 2004, Lecture Notes in Computer Science, vol. 3305, pp. 444-453. Springer-Verlag (2004)

41. Smallbone, K., Gatenby, R., Gillies, R., Maini, P., Gavaghan, D.: Metabolic changes during carcinogenesis: Potential impact on invasiveness. Journal of Theoretical Biology 244, 703713 (2007)

42. Spiro, P., Parkinson, J., Othmer, H.: A model of excitation and adaptation in bacterial chemotaxis. Proceedings of the National Academy of Sciences USA 94, 7263-7268 (1997)

43. Stevens, A.: The derivation of chemotaxis equations as limit dynamics of moderately interacting stochastic many-particle systems. SIAM Journal on Applied Mathematics 61, 183-212 (2000)

44. Sumpter, D.: The principles of collective animal behaviour. Philosophical Transactions of The Royal Society B: Biological Sciences 361, 5-22 (2006)

45. Sumpter, D.: Collective Animal Behavior. Princeton University Press (2010)

46. Wand, M., Jones, M.: Kernel smoothing. Chapman \& Hall/CRC (1994)

47. Wiener, N.: Extrapolation, Interpolation, and Smoothing of Stationary Time Series. MIT Press (1964)

48. Wooldridge, M.: An introduction to multi-agent systems. Wiley (2002) 
49. Xue, C., Budrana, E., Othmer, H.: A hybrid model that explains radial and spiral stream formation in Proteus mirabilis colonies (2011). Submitted

50. Xue, C., Hwang, H.J., Painter, K., Erban, R.: Travelling Waves in Hyperbolic Chemotaxis Equations. Bulletin of Mathematical Biology 1, 1-29 (2010)

51. Xue, C., Othmer, H.: Multiscale models of taxis-driven patterning in bacterial populations. SIAM Journal on Applied Mathematics 70(1), 133-167 (2009) 



\section{RECENT REPORTS}

63/10 A dual porosity model of nutrient uptake by root hairs soil

Zygalakis

Kirk

Jones

Roose

Wissuwa

64/10 Hot Charge Pairs and Charge Generation in Donor Acceptor

Kirkpatrick Blends

65/10 Excluded-volume effects in the diffusion of hard spheres

Bruna

Chapman

66/10 Dynamics of colloidal particles in ice

Spannuth

Mochrie

Peppin

Wettlaufer

01/11 Improving the efficiency of optical coherence tomography by using the non-ideal behaviour of a polarising beam splitter

Lippok

Nielsen

Vanholsbeeck

02/11 Self-diffusion in remodelling and growth

Epstein

Goriely

03/11 Spontaneous rotational inversion in Phycomyces

Goriely

Tabor

04/11 From individual to collective behaviour of coupled velocity jump processes: a locust example

Erban

Haskovec

05/11 Solving Eigenvalue problems on curved surfaces using the closest point method

MacDonald

Brandman

Ruuth

06/11 A numerical methodology for the Painleve equations

Fornberg

Weideman

07/11 Strong stability preserving two-step Runke-Kutta methods

Ketcheson

Gotlieb

MacDonald

08/11 Hysteresis and Post Walrasian Economics

Cross

McNamara

Kalachev

Pokrovskii

09/11 A locally adaptive time-stepping algorithm for petroleum reservoir simulations

McNamara

Bowen

Dellar

10/11 On the predictions and limitations of the BeckerDoring model for reaction kinetics in micellar surfactant solutions

Griffiths

Bain

Breward

Colegate

Howell

Waters 
13/11 Quasi-steady state analysis of two-dimensional random intermittent search processes

Bressloff

Newby

14/11 A Constrained Approach to Multiscale Stochastic Simulation of

Cotter Chemically Reacting Systems

Zygalakis

Kevrekidis

Erban

15/11 The Two Regime Method for optimizing stochastic reaction-

Flegg diffusion simulations

Chapman

Erban

16/11 Recombination via tail states in polythiophene:fullerene solar cells Kirchartz

Pieters

Kirkpatrick

Rau

Nelson

17/11 Energy versus electron transfer in organic solar cells: a comparison

Soon of the photophysics of two indenofluorene: fullerene blend films

Clarke

Zhang

Agostinelli

Kirkpatrick

Dyer-Smith

McCulloch

Nelson

Durrant

18/11 Asymptotic analysis of a pile-up of edge dislocation Hall

19/11 A perturbation analysis of spontaneous action potential initiation

Keener1 by stochastic ion channels

Newby

Copies of these, and any other OCCAM reports can be obtained from:

Oxford Centre for Collaborative Applied Mathematics Mathematical Institute

24 - 29 St Giles'

Oxford

OX1 3LB

England

www.maths.ox.ac.uk/occam 